\title{
Osteosíntesis Bioreabsorbible Bioactivo (Hidroxiapatita/ Poly L-lactido) en Osteotomía Le Fort I Segmentada
}

\author{
Bioresorbable Bioactive Osteosynthesys (Hidroxiapatite / Poly L-lactide) \\ in Le Fort I Segmented Osteotomy
}

Sergio Olate ${ }^{1,2}$; Alejandro Unibazo²; Francisca Uribe ${ }^{1,3}$; Juan Pablo Alister ${ }^{1,3}$ \& Felipe Martínez $z^{1,3,4}$

OLATE, S.; UNIBAZO, A.; URIBE, F.; ALISTER, J. P. \& MARTINEZ, F. Osteosíntesis bioreabsorbible bioactivo (Hidroxiapatita / Poly L-lactido) en osteotomía Le Fort I. Int. J. Odontostomat., 12(2):137-141, 2018.

RESUMEN: El objetivo de este articulo es presentar un caso clínico con la utilización de un innovador sistema de osteosíntesis basado en la mezcla de hidroxiapatita (HA) y acido poliláctico (PLLA). La paciente fue admitida en el Hospital Abraham González Peña de Lautaro (Chile) debido a una deformidad facial que exigió realizar una cirugía ortognática monomaxilar para avance y aumento transversal del maxilar; se utilizaron placas de tipo $L$ y tornillos de HA/PLLA de $5 \mathrm{~mm}$ para la fijación maxilar, realizando la cirugía sin complicaciones; el proceso técnico y alta hospitalaria se ejecutó de forma convencional. Se realizó un análisis de la literatura donde se analiza la versatilidad, la seguridad y la estabilidad de segmentos cuando se utiliza este innovador material y se concluye que este tipo de osteosíntesis es aplicable a cirugía maxilofacial, exigiendo una curva de aprendizaje por parte del cirujano, necesaria para obtener resultados adecuados.

PALABRAS CLAVE: hidroxiapatita, placa reabsorbible, osteosíntesis osteoconductiva.

\section{INTRODUCCIÓN}

El uso de miniplacas y tornillos en cirugía maxilofacial modificó sustancialmente la forma de definir la estabilidad post quirúrgica, mejorando las condiciones para la reparación ósea y la estabilidad a largo plazo. Actualmente, existen diferentes modelos, técnicas y formas de manipulación de la osteosíntesis, optimizando los resultados y la versatilidad de la cirugía (Olate et al., 2010; Andrade et al., 2014; Pozzer et al., 2014; Meram et al., 2018).

Las aleaciones metálicas utilizadas, basadas en el titanio, han sido la norma en cirugía maxilofacial, donde ventajas como la facilidad de manipulación y el costo han permitido su constante aplicación. Por otro lado, los sistemas de osteosíntesis absorbibles se han introducido en el mercado con la ventaja de ofrecer similares condiciones y respuestas a la carga mecánica, considerando su reabsorción y degradación en tiempos variables con la consecuente ventaja de eliminar el metal de la osteosíntesis (Yang et al. 2013; Rodríguez-Chessa et al., 2014).
Desventajas observadas en estos materiales han sido asociadas al tamaño de los tornillos, generalmente de mayor diámetro al comparar con sus similares de titanio, y a la manipulación intraoperatoria que exige el uso de elementos extras (agua caliente, por ejemplo) que permitan alcanzar la flexibilidad de las placas y con ello doblar y modelar el sistema que se debe instalar (Ueki et al., 2011, 2012, 2013).

Los sistemas basados en hidroxiapatita (HA) y ácido poliláctico (PLLA) han mejorado significativamente algunas de estas limitaciones, introduciendo la flexibilidad a la placa sin necesidad de ningún tipo de sustancia intraoperatoria para la manipulación y ha permitido la disminución del tamaño del tornillo, optimizando el procedimiento intraoperatorio y la estabilidad postoperatoria (Ueki et al., 2012). El objetivo de este articulo es presentar el uso de este tipo de osteosíntesis en cirugía ortognática y discutir sus potenciales aplicaciones.

\footnotetext{
${ }^{1}$ División de Cirugía Oral, Facial y Maxilofacial, Universidad de La Frontera, Temuco, Chile

2 Unidad de Cirugía Maxilofacial, Hospital Abraham González Peña, Lautaro, Chile.

${ }^{3}$ Unidad de Cirugía Maxilofacial, Hospital Hernán Henríquez Aravena, Temuco, Chile.

${ }^{4}$ Magister en Odontología, Universidad de La Frontera, Temuco, Chile.
} 


\section{CASO CLINICO}

K.E., paciente de sexo femenino, fue admitida en el Hospital Abraham González Peña de Lautaro (Chile) bajo el diagnóstico de deformidad facial y anomalía dento esqueletal de clase III. La paciente no presentaba alteraciones sistémicas o contraindicaciones para el tratamiento de forma que fue sometida a la fase de ortodoncia pre quirúrgica para posteriormente realizar una cirugía monomaxilar de avance mediante osteotomía de Le Fort I.

En la fase de planificación quirúrgica se determinó un overjet negativo de $-3 \mathrm{~mm}$ junto a una defi-

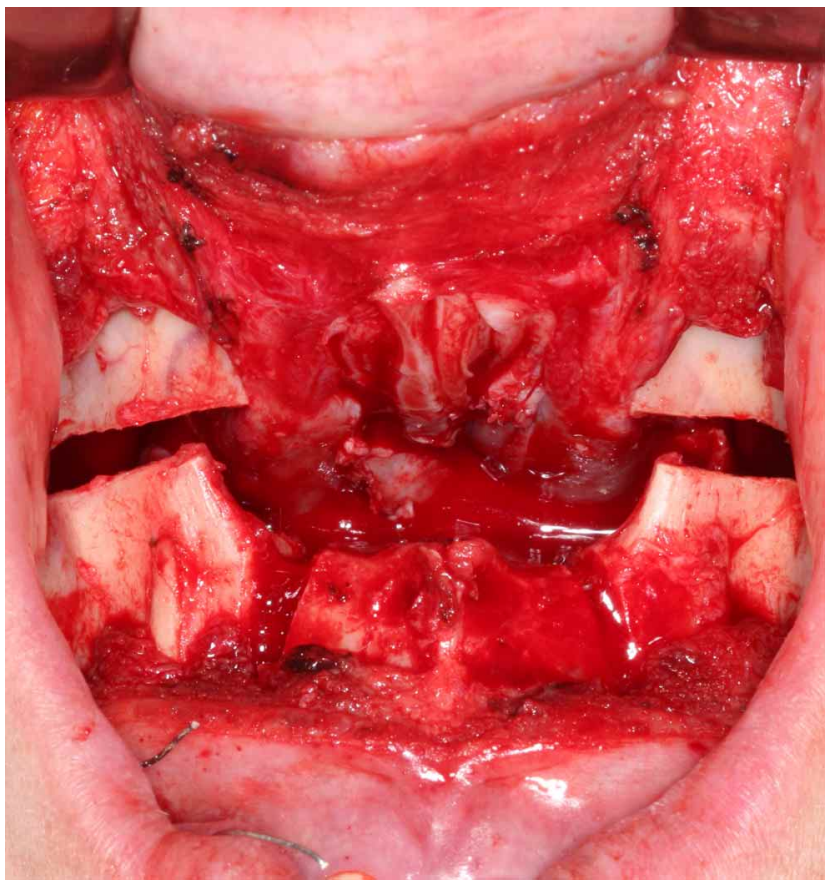

Fig. 1. Osteotomía Le Fort I segmentada entre canino e incisivo lateral. Procedimiento estándar para el posicionamiento en cirugía ortognática

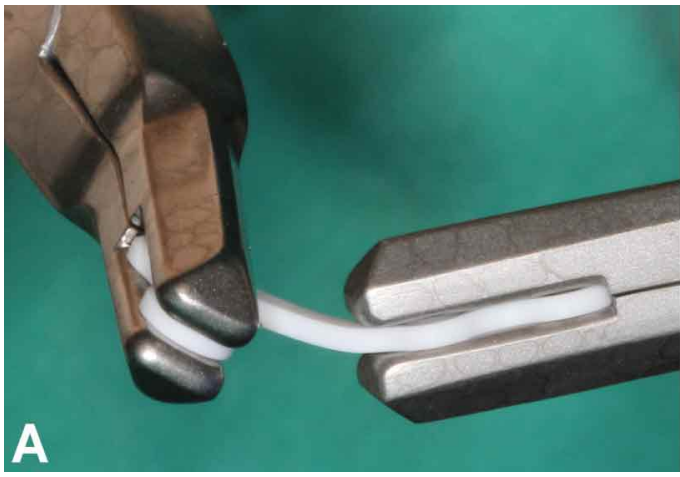

ciencia transversal de $5 \mathrm{~mm}$ lo que permitió la instalación de la técnica de segmentación maxilar en conjunto con el avance maxilar propuesto (Fig. 1).

\section{El sistema de osteosíntesis OsteotransTM} (Takiron Co., Osaka, Japón) consiste de placas y tornillos presentados en envoltorios individuales, con un proceso de esterilizado dependiente del fabricante (no es posible la esterilización en le hospital) (Fig. 2). Las placas seleccionadas fueron de tipo "L" considerando la planificación de avance de $5 \mathrm{~mm}$ y la expansión transversal de $6 \mathrm{~mm}$. Una gentil manipulación de las placas fueron realizadas para conseguir curvar la placa sin generar un estrés importante en el material (Fig. 3 a,b); la modelación de la placa se realizó lentamente hasta alcanzar la forma deseada ya que el fabricante indica límites para la magnitud de doblado probable de alcanzar, estableciendo un límite de doblado.

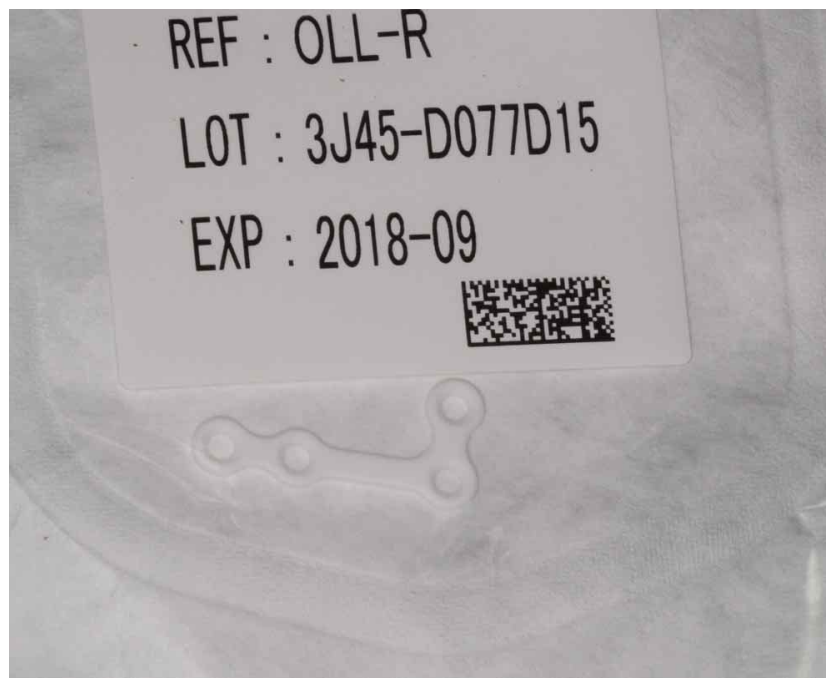

Fig. 2. Embalaje utilizado por la empresa para la entrega del material bioactivo estéril; cada elemento se presenta en envoltorios individuales.

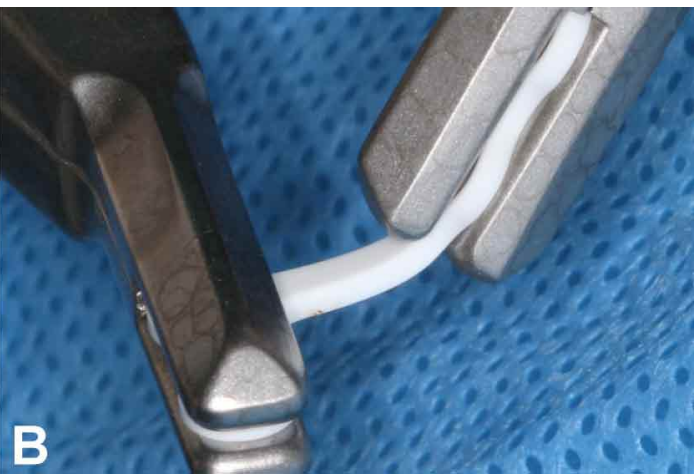

Fig. 3. Doblado y manipulación de la placa de HA/PLLA; el sistema exige que el doblado sea realizado con dobladores específicos; existe límite para la manipulación de las placas. 


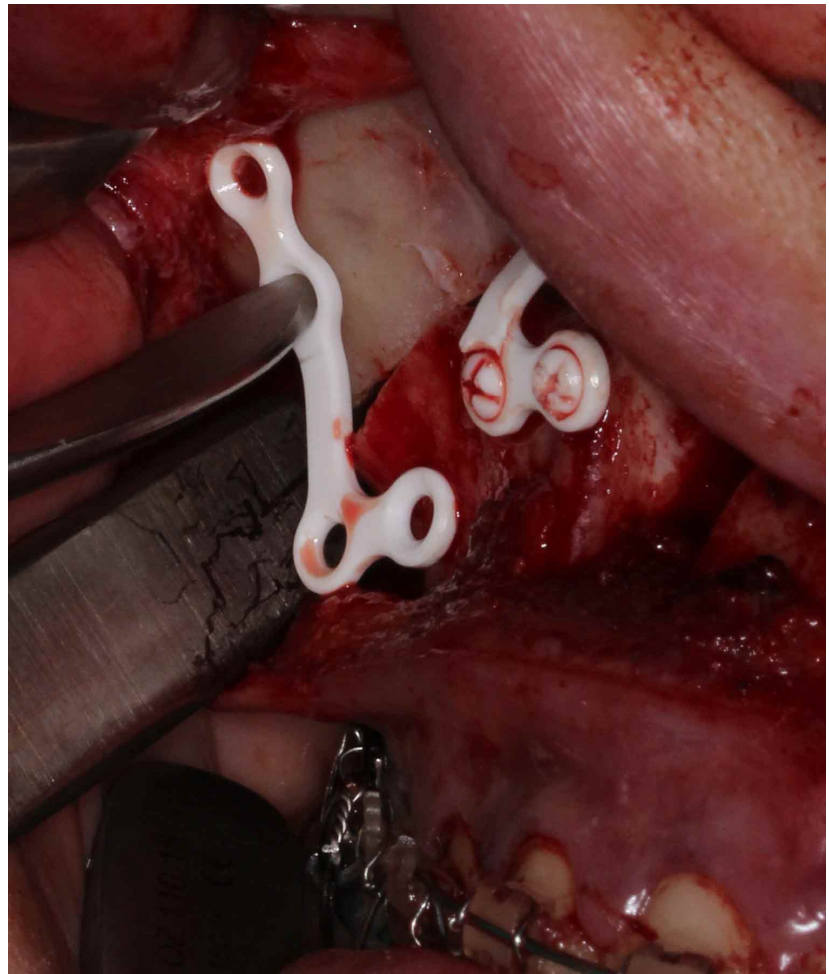

Fig. 4. Posicionamiento de la placa distal a nivel del pilar zigomático maxilar; la ubicación y modelado se realiza de forma convencional. OsteotransTM (Takiron Co., Osaka, Japón) presenta también sistemas de placas pre dobladas que pueden facilitar algunas etapas.

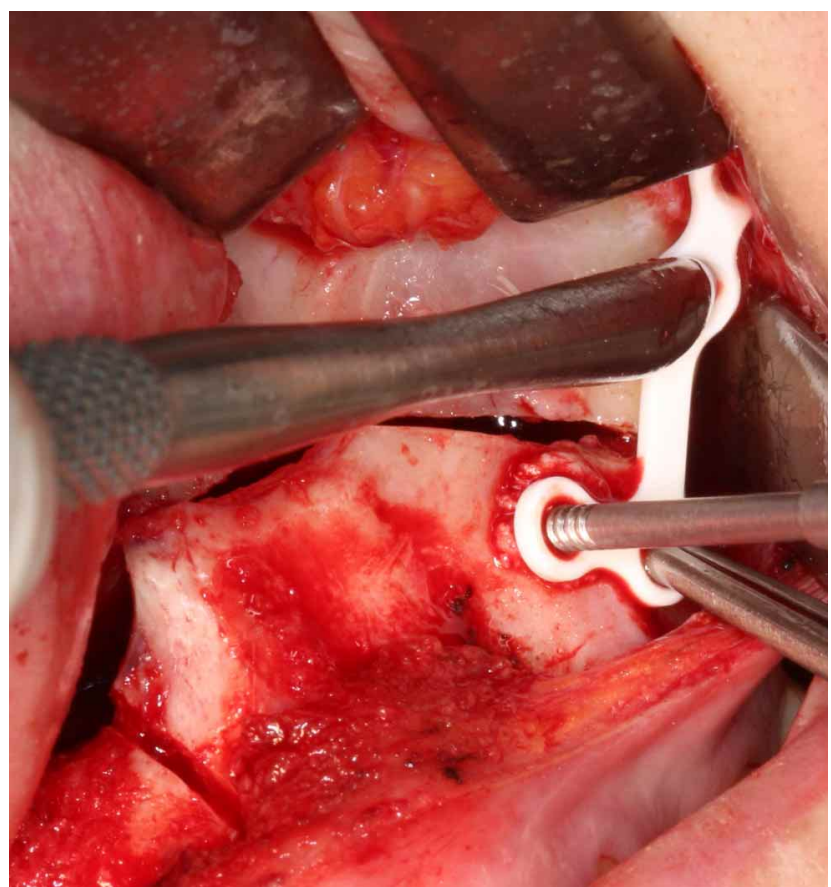

Fig. 5. El conformador de rosca debe ser utilizado después de la perforación con brocas; el uso es aplicado a todas las perforaciones que requieran tornillos de HA/PLLA
Una vez modelada la placa, fue instalada y estabilizada con tornillos del mismo material (Fig. 4); el sistema de fresado utilizado no presenta diferencia al realizado con los clásicos sistemas de titanio; luego del fresado es necesario que un dispositivo conformador de rosca sea utilizado para permitir la instalación de los tornillos sin inconvenientes (Fig. 5); el control de la fuerza en esta etapa es necesaria para no modificar la cabeza del tornillo que es susceptible de modificación cuando se aplica una fuerza exagerada (Fig. 6). La fijación total de la maxila con 4 placas

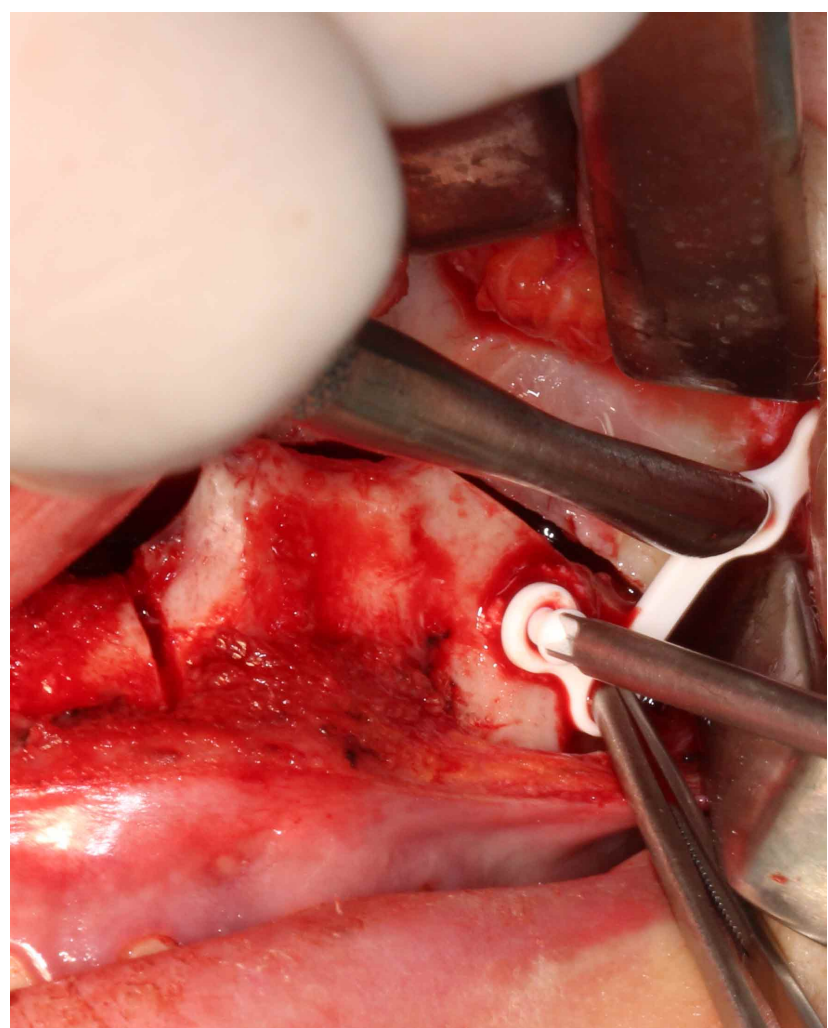

Fig. 6. La instalación del tornillo exige un control de fuerza en la instalación para no alterar la cabeza normal del tornillo de HA/PLLA

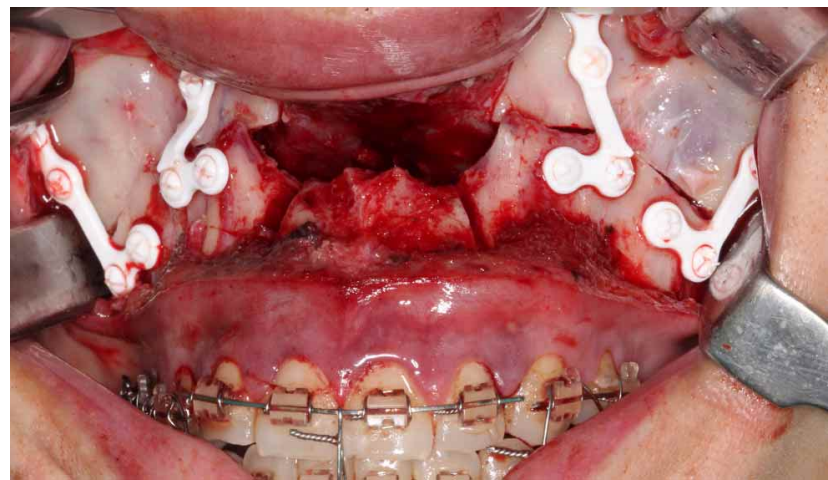

Fig. 7. Osteosíntesis con 4 placas de tipo L para la fijación de la osteotomía Le Fort I. 
de osteosíntesis se realizó sin restricciones y permitió la estabilización de la maxila de forma adecuada (Fig. 7). El seguimiento postoperatorio mostró estabilidad de la osteotomía y del posicionamiento maxilar.

\section{DISCUSIÓN}

Existe evidencia de que los materiales de osteosíntesis reabsorbible tienen adecuada respuesta al estrés y mantienen estabilidad a mediano y largo plazo; Yang et al. presentaron una revisión sistemática con 1092 pacientes incluidos, demostrando que los sistemas de fijación de tipo absorbible, basados en PLLA, presentan una estabilidad adecuada aunque podrían presentar mayor tasa de complicaciones al comparar con los materiales metálicos.

A pesar de que van Bakalen et al. (2015) concluyeron que el uso de materiales metálicos puede ser mejor desde el punto de vista de costo y efectividad en cirugía maxilofacial convencional, materiales reabsorbibles aumentan su presencia en cirugías ya que pueden ser especialmente útiles en el tratamiento de fracturas y osteotomías en pacientes pediátricos (Muñante-Cárdenas et al., 2011); pacientes que no desean metal, pacientes con alergia o pacientes que deberán ser sometidos a futuros exámenes en la región cráneo máxilo facial también son candidatos al uso de materiales absorbibles.

La HA es un componente inorgánico con posibilidades de calcinado a $800-900{ }^{\circ} \mathrm{C}$ y sinterizado a $1000-1400{ }^{\circ} \mathrm{C}$, presentando una composición semejante al hueso. Este sistema innovador de osteosíntesis (HA/PLLA) ha sido utilizado en cirugía traumatológica y en cirugía maxilofacial (Yasunaga et al., 1999; Matsumoto et al., 2002); tiene la ventaja, a diferencia de las placas a base de PLLA, de visualizarse en cualquier estudio de imagen, lo que implica un fácil reconocimiento del material en caso de fractura de la placa o movilización de la misma. Presenta además biocompatibilidad y confirmada intervención en la osteoconducción de la zona de instalación (Furukawa et al., 2000).

Ueki et al. (2012) presentaron una secuencia de 60 pacientes tratados con osteotomía de Le Fort I, donde 20 sujetos fueron tratados con placas a base de uHA/PLLA de tipo L, en 20 sujetos se utilizo PLLA tipo $L$ y en los últimos 20 se utilizó placas de titanio tipo L; en el seguimiento no se observaron infeccio- nes, inestabilidad ósea y en el largo plazo no existió diferencias en términos de recidiva de la cirugía, estableciendo una oclusión favorable en los tres grupos. Posteriormente, utilizando similar metodología, Ueki et al. (2013) compararon la estabilidad de la fijación de la osteotomía de Le Fort I utilizando placas de uHA/PLLA, donde evaluaron diferentes movimientos de reposición superior, inferior, o avance maxilar, utilizando también tricalcio fosfato como relleno en los espacios generados en las osteotomías; no se reportaron infecciones o complicaciones y en los estudios cefalométricos se observó estabilidad en la posición de la maxila, indicando algunas limitaciones dependiendo de algunos movimientos mas exigentes de la maxila. Similares conclusiones obtuvieron Landes et al. (2014), quienes realizaron un estudio comparativo de la fijación con HA/PLLA y la fijación con titanio en cirugía de Le Fort I, sin encontrar diferencias significativas en la estabilidad de ambos grupos.

El presente caso fue tratado con osteotomía de Le Fort I y segmentación maxilar, procedimiento estándar y exigente en la reposición y osteosíntesis de segmentos (Olate et al., 2014); la cirugía y la posterior estabilidad se realizó sin complicaciones, aunque el empleo de este tipo de osteosíntesis implica asumir una curva de aprendizaje del equipo quirúrgico, realizando el control de fuerza en el doblado de la placa y la correcta manipulación de los tornillos en el proceso de fijación.

Este sistema de placas también ha sido utilizada en otras condiciones quirúrgicas; en cirugía mandibular con osteotomía sagital de rama y retrusión mandibular, Ueki et al. (2011) demostraron la eficiencia del sistema de placas HA/PLLA, que resulto similar a las placas de PLLA y las placas de titanio.

En trauma maxilofacial, Landes et al. utilizaron placas basadas en HA/PLLA para reducción y osteosíntesis en fracturas de hueso zigomático, del tercio medio y superior facial, interviniendo 29 sujetos; concluyeron que este sistema presentaban una adecuada estabilidad, en ausencia de complicaciones intraoperatorias; también reportaron en estudios histológicos posteriores la neoformación ósea en contacto intimo con el material de osteosíntesis implantado. Hayashi et al. (2013) también estudiaron 17 casos de fracturas múltiples faciales tratadas con este sistema de osteosíntesis (OsteotransTM, Takiron Co., Osaka, Japón), reportando estabilidad y eficacia en el tratamiento; también indicaron menores complicaciones asociadas al proceso inflamatorio observado en una zona fracturada. 
En base a nuestra experiencia inicial y los reportes de la literatura, podemos concluir que los sistemas de osteosíntesis basados en HA/PLLA son seguros y eficientes en la aplicación clínica; los estudios demuestran adecuados resultados en términos de estabilidad a corto y mediano plazo, aunque la curva de aprendizaje debe ser realizada por los cirujanos cuando comienzan con los procesos de manipulación con este material.

OLATE, S.; UNIBAZO, A.; URIBE, F.; ALISTER, J.P.; MARTINEZ, F. Bioresorbable bioactive osteosynthesys (Hidroxiapatite / Poly L-lactide) in Le Fort I segmented osteotomy. Int. J. Odontostomat., 12(2):137-141, 2018.

ABSTRACT: The aim of this paper is to show a clinical case, where was used an innovative osteosynthesys system based on the mixture of hidroxiapatite (HA) and polilactic acid (PLLA). The patient was admitted into Abraham González Peña Hospital (Chile), showing a facial deformity to treat with monomaxillary orthognathic surgery for advance and transversal increase of the maxilla.; were used type $L$ plates and $5 \mathrm{~mm}$ screws of HA/PLLA for the internal fixation, performing the surgery without complications; the technical process and the ALTA hospital was realized in a conventional strategy. Was realized a literature review showing the versatility, safe and stability of bone segment when used this innovative material and it's concluded that this osteosynthesis system can be used normally in maxillofacial surgery; a learning curve to learn about this material is needed by surgeons.

KEY WORDS: hydroxyapatite, absorbable plate, osteoconductive osteosynthesis.

\section{REFERENCIAS BIBLIOGRÁFICAS}

Andrade, V. C.; Luthi, L. F.; Sato, F. L.; Nascimento, F. F.; Olate, S. \& Albergaria-Barbosa, J. R. Sagittal split ramus osteotomy with $L$ inverted osteosynthesis. A mechanical study with photoelasticity. Int. J. Odontostomat., 8(1):161-5, 2014.

Furukawa, T.; Matsusue, Y.; Yasunaga, T.; Nakagawa, Y.; Okada, Y.; Shikinami, Y.; Okuno, M. \& Nakamura, T. Histomorphometric study on high-strength hydroxyapatite/poly(L-lactide) composite rods for internal fixation of bone fractures. J. Biomed. Mater. Res., 50(3):410-9, 2000.

Hayashi, M.; Muramatsu, H.; Sato, M.; Tomizuka, Y.; Inoue, M. \& Yoshimoto, S. Surgical treatment of facial fracture by using unsintered hydroxyapatite particles/poly l-lactide composite device (OSTEOTRANS MX(®)): a clinical study on 17 cases. $J$. Craniomaxillofac. Surg., 41(8):783-8, 2013.

Landes, C. A.; Ballon A.; Tran, A.; Ghanaati, S. \& Sader, R. Segmental stability in orthognathic surgery: hydroxyapatite/Poly-I-lactide osteoconductive composite versus titanium miniplate osteosyntheses. J. Craniomaxillofac. Surg., 42(6):930-42, 2014.
Matsumoto, M.; Chosa, E.; Nabeshima, K.; Shikinami, Y. \& Tajima, $\mathrm{N}$. Influence of bioresorbable, unsintered hydroxyapatite/poly-Llactide composite films on spinal cord, nerve roots, and epidural space. J. Biomed. Mater. Res., 60(1):101-9, 2002.

Meram, A. T.; Olate, S. \& Palmieri, C. F. Jr. Is the three-dimensional strut plate an adequate fixation technique for mandibular symphysis fractures? J. Oral Maxillofac. Surg., 76(1):140-5, 2018.

Muñante-Cárdenas, J. L.; Olate, S.; Asprino, L.; de Albergaria Barbosa, J. R.; de Moraes, M. \& Moreira, R. W. Pattern and treatment of facial trauma in pediatric and adolescent patients. J. Craniofac. Surg., 22(4):1251-5, 2011.

Olate, S.; Lima, S. M. Jr.; Sawazaki, R.; Moreira, R. W. \& de Moraes, M. Surgical approaches and fixation patterns in zygomatic complex fractures. J. Craniofac. Surg., 21(4):1213-7, 2010.

Olate, S.; Pozzer, L.; Unibazo, A.; Huentequeo-Molina, C.; Martinez, F. \& de Moraes, M. LeFort I segmented osteotomy experience with piezosurgery in orthognathic surgery. Int. J. Clin. Exp. Med., 7(8):2092-5, 2014

Pozzer, L.; Olate, S.; Cavalieri-Pereira, L.; de Moraes, M. \& Albergaría-Barbosa, J. R. Influence of the design in sagittal split ramus osteotomy on the mechanical behavior. Int. J. Clin. Exp. Med., 7(5):1284-8, 2014.

Rodríguez-Chessa, J.; Olate, S.; Netto, H. D.; Noia, C.; de Moraes, M. \& Mazzonetto, R. In vitro resistance of titanium and resorbable (poly L-co-DL lactic acid) osteosynthesis in mandibular body fracture. Int. J. Oral Maxillofac. Surg., 43(3):362-6, 2014.

Ueki, K.; Okabe, K.; Marukawa, K.; Mukozawa, A.; Moroi, A.; Miyazaki, M.; Sotobori, M.; Ishihara, Y.; Yoshizawa, K.; Ooi, K. \& Kawashiri, S. Maxillary stability after Le Fort I osteotomy with self-setting a-tricalcium phosphate and an absorbable plate. Int. J. Oral Maxillofac. Surg., 42(5):597-603, 2013.

Ueki, K.; Okabe, K.; Miyazaki, M.; Mukozawa, A.; Moroi, A.; Marukawa, K.; Nakagawa, K. \& Yamamoto, E. Skeletal stability after mandibular setback surgery: comparisons among unsintered hydroxyapatite/poly-L-lactic acid plate, poly-L-lactic acid plate, and titanium plate. J. Oral Maxillofac. Surg., 69(5):1464-8, 2011.

Ueki, K.; Okabe, K.; Moroi, A.; Marukawa, K.; Sotobori, M.; Ishihara, Y. \& Nakagawa, K. Maxillary stability after Le Fort I osteotomy using three different plate systems. Int. J. Oral Maxillofac. Surg., 41(8):942-8, 2012.

Yang, L.; Xu, M.; Jin, X.; Xu, J.; Lu, J.; Zhang, C.; Tian, T. \& Teng, L. Complications of absorbable fixation in maxillofacial surgery: a meta-analysis. PLoS One, 8(6):e67449, 2013.

Yasunaga, T.; Matsusue, Y.; Furukawa, T.; Shikinami, Y.; Okuno, M. \& Nakamura, T. Bonding behavior of ultrahigh strength unsintered hydroxyapatite particles/poly(L-lactide) composites to surface of tibial cortex in rabbits. J. Biomed. Mater. Res., 47(3):412-9, 1999.

Dirección para correspondencia:

Prof. Dr. Sergio Olate

División de Cirugía Oral, Facial y Maxilofacial

Centro de Excelencia en Estudios Morfológicos y Quirúrgicos

Universidad de La Frontera

Temuco - CHILE

E-mail: sergio.olate@ufrontera.cl

Recibido : 04-02-2018

Aceptado: 07-05-2018 